\title{
Sinn und Unsinn einer Kopenhagen-Kommission
}

verfassungsblog.de/sinn-und-unsinn-einer-kopenhagen-kommission/

Claudio Franzius Mo 8 Apr 2013

Mo 8 Apr 2013

\section{Versäumnisse}

Von den politischen Akteuren wird eingeräumt, dass es ein Fehler war, auf die Erfüllung der KopenhagenKriterien für den Beitritt zu drängen, nach dem erfolgreichen Beitritt aber keine Evaluierungsmechanismen für die Überprüfung der dauerhaften Einhaltung der Kriterien in der Hand zu haben. Das könnte durch die Einrichtung einer Kopenhagen-Kommission, verstanden als watchdog, die auf das Abgleiten in einen kruden Nationalismus à la Ungarn aufmerksam macht, nachgeholt werden.

Richtig ist auch, dass wir von der „nuklearen Option“ des Art. 7 EUV nicht zu viel erwarten können. Dass dieses Verfahren keinen Ausschluss aus der Union vorsieht, hat allerdings gute Gründe, die dem Charakter der EU als freiwilligem Zusammenschluss von Staaten geschuldet sind. Die Union ist keine Zwangsgemeinschaft, sondern eine Rechtsgemeinschaft. Auch ein Ausschluss aus dem Euro ist, daran darf erinnert werden, in den Verträgen so nicht vorgesehen. Ein Staat müsste vielmehr, auf Zureden der Anderen, zur Einleitung des Austrittsverfahrens aus der EU bewogen werden. Skandalös ist weniger, wie Müller in seinem Essay meint, die fehlende Ausschlussmöglichkeit als vielmehr das systematische Wegschauen der nationalen Regierungen, dem durch eine neue Institution nicht abzuhelfen sein und von der sich eine integrationsunwillige Regierung kaum beeindrucken lassen dürfte.

Es ist eben auch Sache der nationalen Akteure, wie mit Europa umgegangen wird. An einen Ausschluss der ungarischen Fidesz aus der EVP-Fraktion oder der rumänischen Sozialliberalen Union (USL) aus der SPEFraktion wird man solange nicht denken können, solange die europäischen Parteien im Europäischen Parlament nur als Ableger der nationalen Parteien fungieren und keine größere Eigenständigkeit erhalten haben.

\section{Demokratie: Zusammenhänge und Optionen}

Es verdient mit Müller festgehalten zu werden, dass das Intaktbleiben der mitgliedstaatlichen Demokratien eine europäische Frage ist. Weil die EU einen Teil ihrer Legitimation aus den Mitgliedstaaten bezieht, ist sie auf das Funktionieren der mitgliedstaatlichen Demokratien angewiesen. Oder anders gesagt: Will die Union demokratisch sein, können ihr die demokratisch verfassten Mitgliedstaaten nicht gleichgültig sein. Diese Frage zurückzustellen, könnte sich für Europa als verheerend erweisen. Wo es um den Erhalt einer freiheitlichdemokratisch verfassten Struktur in den Mitgliedstaaten geht, sehen die politischen Maßnahmen der Unionsorgane bislang eher dürftig aus und es entsteht eine gefährliche Schieflage, einerseits über die deutscheuropäische Sparpolitik in der Eurokrise reihenweise demokratisch gewählte Regierungen aus dem Amt zu jagen, andererseits massiven Verschlechterungen der innerstaatlichen Demokratie nicht nur am Rand (Ukraine), sondern im Herzen Europas (Ungarn) tatenlos zuzuschauen.

Doch welche Optionen gibt es? Unterschieden werden kann zwischen kurzfristigen und langfristigen Maßnahmen: Die Kommission begnügt sich mit dem traditionellen Vorgehen, punktuell Vertragsverletzungen zu rügen. Langfristig ausgelegte Strategien zum Schutz der mitgliedstaatlichen Demokratien sind demgegenüber nicht ersichtlich. Abhilfe könnte eine den bestehenden Verfahren vorgeschaltete Kopenhagen-Kommission sein, die, wie Müller empfiehlt, ein Auge auf alle Mitgliedstaaten wirft und rechtzeitig „politischen Alarm“ schlägt.

Zu unterscheiden ist aber auch zwischen in die Logik des Integrationsverbundes gestellten Möglichkeiten und neuen Verfahren der Demokratiesicherung, die gegebenenfalls einer Vertragsänderung bedürfen: Die EU als nicht-hierarchische Ordnung basiert auf der freiwilligen Befolgung des europäischen Rechts. Nicht Zwang, sondern die wechselseitige Anerkennung der Rechte und Pflichten ist die Grundlage des europäischen Projekts. Das spricht dafür, weiterhin auf dezentrale Mechanismen der Rechtsdurchsetzung zu setzen, um die Selbstbefähigung mitgliedstaatlicher Institutionen zur Formulierung einer europäischen Perspektive zu stärken. 
Erst das erlaubt es, die europäischen Werte nicht als fremde, sondern als die eigenen Werte zu akzeptieren. Müller sieht die Gefahr des Paternalismus, sollen Unionsorgane politisch intervenieren, meint dies aber hinnehmen zu können, gebe es doch in der Union als politischer Gemeinschaft keine allein nationalen Angelegenheiten mehr. Das ist richtig, kann aber nicht darüber hinwegtäuschen, dass es für die Einrichtung einer politisch wirkungsvollen Instanz zur Demokratiesicherung einer - wenig wahrscheinlichen Vertragsänderung bedarf.

Weil die Eigenlegitimation unionaler Herrschaftsgewalt nicht staatsanalog konstruiert werden kann, die EU vielmehr auf funktionierende politische Systeme in den Mitgliedstaaten angewiesen bleibt, müssen Vorschläge die spezifische Verbundstruktur in der Verzahnung der Ebenen im Blick behalten, aber auch das wechselseitige Zusammenspiel von Recht und Politik in der Mehrebenendemokratie. In diesem Zusammenhang ist die Frage wichtig, wer zum Schutz der Demokratie berufen sein soll. Soweit individuelle Rechte in Frage stehen, wird den Gerichten eine besondere Verantwortung für die Wahrung der Grundrechte nicht abgesprochen werden können. Das gilt für nationale wie für europäische Gerichte. Zwar lässt sich die strukturelle Schwäche politischer Maßnahmen nur begrenzt durch juristische Lösungen kompensieren. Politische und rechtliche „Problemlösungsangebote" stehen jedoch in keinem einfachen Alternativverhältnis: Politische Lösungen, wie sie Müller einfordert, haben die hohen Opportunitätskosten in Rechnung zu stellen, werden eine Beschäftigung der Gerichte aber kaum entbehrlich machen können. Demgegenüber haben rechtliche, von den Unionsbürgern vor dem nationalen Gericht einklagbare Lösungen die Grenzen eines über die Sicherung der Menschenrechte hinausgehenden judikativen Demokratieschutzes zu beachten.

\section{Recht und Politik}

Diesen Punkt vernachlässigt Müller. Sein Vorschlag einer Kopenhagen-Kommission ist weder das eine noch das andere, weder eine politische Lösung noch rechtlich überzeugend. Einerseits, so heißt es bei inm, würden durch den bestehenden Exekutivföderalismus politische Optionen versperrt, andererseits sei ein umgekehrter Solange-Vorbehalt zu legalistisch und unpolitisch, weil auf die Unionsbürgerschaft abgestellt wird. Dem ist zu widersprechen. Es reicht nicht aus, den - aus dem deutschen Bundesstaat bekannten - Exekutivföderalismus der EU mit Jürgen Habermas zu verdammen, spiegelt er doch jene demokratischen Verschränkungen unter dem Erhalt der mitgliedstaatlichen Demokratien, welche die Union in struktureller Hinsicht auszeichnen. Auch kann es in der europäischen Rechtsgemeinschaft nicht um ein gegenseitiges Ausspielen von Recht und Politik gehen. Dass für politische Probleme politische Lösungen zu suchen sind, ist unbestritten. Auch lässt sich kaum bezweifeln, dass eine Politisierung der Union notwendig ist. Dass es aber das Recht sein muss, dass die Schwelle für politische Interventionen in die mitgliedstaatlichen Demokratien angibt, wird man nicht bestreiten können. „Politischer Alarm“ darf nicht blind und maßstabslos erfolgen. Es spricht vieles dafür, sich an der systematischen Verletzung der grundlegenden Werte, wie sie in Art. 2 EUV niedergelegt sind, zu orientieren.

Unsicher ist, inwieweit die Einhaltung dieser Werte eine Voraussetzung für die Inanspruchnahme der nationalen Verfassungsidentität nach Art. 4 II EUV ist. Die hieran unter Aktivierung der Ruiz Zambrano Rechtsprechung anknüpfende Umkehrung des Solange-Vorbehalts ist aber keine zentralistische, die Trennwand zwischen EURecht und nationalem Recht einreißende Strategie, sondern eingebettet in die Entwicklungszusammenhänge der Unionsrechtsordnung, zu der man stehen mag wie man will, die jedoch zusammen mit den mitgliedstaatlichen Verfassungsordnungen die Grundlage für das politische Projekt der europäischen Integration ist. Auf das Recht als Bindeglied der Gemeinschaft zu setzen, ist nicht idealistisch, sondern realistisch, beruht die EU doch auf keinem kollektiven Makrosubjekt, sondern auf den Bürgern, die freilich imstande sein müssen, ihre Rechte im europäischen Rechtsraum wirksam zur Geltung bringen zu können. Das ersetzt nicht politische Prozesse, ist aber eine dezentrale Strategie, die davon abhängig ist, dass nationale Institutionen den Streit vor den EuGH bringen. Und es löst nicht das Problem eines entmachteten ungarischen Verfassungsgerichts, sind die eigentlichen Adressaten des Vorlageverfahrens doch eher die Fachgerichte, taugt also eher für den Alltag als für politische Konflikte, deren Austragung und Kanalisierung mit gutem Grund - wie es auch Agnes Heller fordert - der ungarischen Opposition überlassen sein könnte.

Wozu also eine Kopenhagen-Kommission als einem Gremium, das bestehende Instrumente nicht ersetzen, aber auf dessen Einsatz drängen können soll? Woher bezöge ein solches Gremium seine Legitimation? Es soll ja 
gerade nicht bloß um die Wahrung individueller Rechte gehen, sondern um das Anstoßen politischer Lösungen. Man wird erhebliche Zweifel anmelden müssen, ob ein „Weisen-Gremium“ besser als die bestehenden Institutionen in der Lage ist, die Probleme beim Namen zu nennen und einer Lösung zuzuführen.

Warum nicht die Europäische Kommission? Müller nennt verräterische Gründe. Man könne nicht beides gleichzeitig haben, also Demokratieschutz und direkt demokratisch legitimierte Institutionen zum Demokratieschutz. Wer die Politisierung der Kommission anstrebe, könne von ihr nicht die überparteiliche Sicherung der Verträge erwarten. Demgegenüber will Müller ein holistisches, die gesamte politische Landschaft erfassendes Quasi-Gericht, dessen Legitimation mehr als prekär wäre. Und dass eine Kopenhagen-Kommission größere Durchschlagskraft als die Venedig-Kommission des Europarates erhielte, kann man bezweifeln, zumal letztere für die Lage in Ungarn mit der Schwächung des Verfassungsgerichts, der übermäßigen Anwendung der Schwerpunktgesetze, der unscharfen Definition der Pressefreiheit und der Minderheitenrechte sowie der zu offenen Beschreibung des Verhältnisses zu den im Ausland lebenden ungarischen Minderheiten wesentliche Kritikpunkte weitgehend folgenlos benannt hat. Es ist richtig, dass wir weniger Begrenzungen und mehr „Empowerment“ benötigen. Bürger und mitgliedstaatliche Institutionen müssen befähigt werden, für die gemeinsam geteilten Werte zu kämpfen. Insoweit kann in der „Rückendeckung“ für die Sicherung der grundlegenden Unionsbürgerrechte eine Bedingung für den politischen Wandel gesehen werden, der von den politischen Kräften vor Ort zu verantworten ist. Die Stärkung der Selbstbefähigung der Bürger, ihre Angelegenheiten als europäische Angelegenheiten vor die Institutionen des Heimatstaates zu bringen, zielt dann auch nicht auf die Ersetzung, sondern auf die Ermöglichung rechtlich umhegter politischer Lösungen.

Und warum eine Institution? Die Einschaltung des EuGH ist keine zentralistische Lösung, sondern Ausdruck der Verbundordnung, die im konstitutionellen Pluralismus politisch gespiegelt wird. Es ist vielleicht kein Zufall, dass Art. 4 II EUV bei Müller keine Erwähnung findet. Damit übersieht er freilich einen wesentlichen Baustein der konstitutionellen Ordnung, die auf einen dezentralen Vollzug der grundlegenden Werte angelegt ist und auch der Lösung über ein erweitertes, aber maßstabsloses Vertragsverletzungsverfahren entgegensteht. Der Vorschlag von Müller ist so gesehen sehr deutsch, erinnert an Erich Lüth und die Wertordnung mit der Sehnsucht nach einer Instanz, von der wir - sei es gerichtlich oder politisch - die Klärung grundlegender Fragen erwarten dürfen. Nichts spricht gegen ein institutionelles Denken, was in der Politikwissenschaft keineswegs selbstverständlich ist. Aber die eine Instanz, ob es nun eine lediglich Empfehlungen aussprechende Kopenhagen-Kommission oder das „letztverbindlich“ entscheidende Verfassungsgericht ist - diese Instanz gibt es im konstitutionellen Pluralismus nicht mehr. Das gilt für die Mitgliedstaaten und ihre Organe ebenso wie für die EU mit neuen Institutionen wie einer Kopenhagen-Kommission, die mehr verspricht als sie einzulösen vermag.

LICENSED UNDER CC BY NC ND

SUGGESTED CITATION Franzius, Claudio: Sinn und Unsinn einer Kopenhagen-Kommission, VerfBlog, 2013/4/08, http://verfassungsblog.de/sinn-und-unsinn-einer-kopenhagen-kommission/. 